\title{
From theories of aging to anti-aging interventions
}

\author{
Alexey A. Moskalev ${ }^{1,2,3 * t}$ and Elena G. Pasyukova ${ }^{4 * t}$ \\ ${ }^{1}$ Radiation Ecology, Laboratory of Molecular Radiobiology and Gerontology, Institute of Biology of Komi Science Center of Ural Branch of RAS, Syktyvkar, Russia \\ ${ }^{2}$ Syktyvkar State University, Syktyvkar, Russia \\ ${ }^{3}$ Moscow Institute of Physics and Technology (State University), Dolgoprudny, Russia \\ ${ }^{4}$ Laboratory of Genome Variation, Institute of Molecular Genetics, Russian Academy of Sciences, Moscow, Russia \\ *Correspondence: amoskalev@list.ru; egpas@rambler.ru \\ t'These authors have contributed equally to this work and co-corresponding authors.
}

Edited and reviewed by:

Blanka Rogina, University of Connecticut Health Center, USA

Keywords: aging, evolution, genetics, epigenetics, geroprotectors

The 2nd International Conference «Genetics of aging and longevity $\gg$ took place 22-25 April, 2012 in Moscow, Russia. Top gerontologists and geneticists from 25 countries around the world discussed the current problems in many areas related to the genetics of longevity and mechanisms of aging. Following this meeting, a collection of articles based on the talks, reports and experimental outcomes related to the topics of the conference was published in Frontiers in Genetics of Aging. This collection represents a comprehensive prospect of recent advances in genetics of aging, ranging from theoretical questions to practical approaches aimed to delay aging and prolong lifespan.

Unraveling the proximal cause of aging and creating a universal aging theory remains a difficult and ambitious task. Complicated mechanisms that preserve homeostasis and prolong lifespan involve interactions of numerous genes, metabolic pathways and environmental cues. Genetic and environmental variations, combinations of programmed and random events underlying the aging process further complicate the objective. Trindade et al. (2013) emphasize that a universal evolutionary aging theory must reconcile "trade-offs (pleiotropy or hitchhiking effect), retrogression (mutational load and drift) and direct adaptation ("program")."

The important notion that aging and lifespan are intimately related to various life history traits is highlighted in several articles from the collection. Novoseltsev and Novoseltseva (2013) used mathematical approaches to assess the origin of stochasticity in Drosophila reproduction, in relation to life expectancy. To add to mathematical predictions, analysis of long-term captive breeding of water voles demonstrated that individuals who began breeding at an older age had a significantly longer lifespan and produced more offspring (Nazarova, 2013).

These results resonate with the basis of the aging theory that set the grounds for a heated debate concerning the proximal cause of aging (Zimniak, 2012). In spite of many new insights into regulatory mechanisms that affect the aging process, the author remains true to the hypothesis of molecular damage and argues that hyper function proposed as a universal cause of aging by Blagosklonny (for references, see Zimniak, 2012) is only one of several sources of molecular damage. It is not proved, however, that a single proximal cause of aging does exist. According to the point of view of Lehmann et al. (2013), telomere length and body temperature are independent drivers of mammalian longevity.
New facts are needed to advance our understanding of aging, and studies of model organisms continue to provide us with valuable information. There are five articles in the collection, which represent new discoveries in genetics of aging of Caenorhabditis elegans (Bharill et al., 2013; Coburn and Gems, 2013), Drosophila melanogaster (Rogina and Helfand, 2013; Shostal and Moskalev, 2013), and mice (Bartke and Westbrook, 2012). In one of the most intriguing articles, Coburn and Gems (2013) come to the conclusion that the blue fluorescence of gut granules, a marker of death in C. elegans, is issued from anthranilic acid glucosyl esters, rather than from lipofuscin. They believe that this removes one reason for believing that worm aging is caused by accumulation of molecular damage, thus entering into the extramural discussion with Zimniak (2012).

In recent years, studies of long-lived wild animals, mainly rodents have complemented studies of model species and open new perspectives in aging research. In particular, the longevity of these species is correlated with cancer resistance, and analysis of molecular mechanisms underlying this property can benefit human health if such mechanisms can be activated in human cells (Azpurua and Seluanov, 2013).

Analysis of human populations represents an invaluable source of information concerning genetic and environmental factors promoting a long and healthy life. The results of several longitudinal studies of centenarians suggest, on the one hand, that factors responsible for exceptional longevity and health are not necessary the same and centenarians often experience chronic age-related diseases, but are able to cope with them. On the other hand, these results suggest that postponing aging changes is associated with extreme longevity (Sebastiani and Perls, 2012; Yashin et al., 2013). To add to the complexity, it is likely that the genetic component of extreme longevity includes many genes with modest effects (Sebastiani and Perls, 2012), which underscores once again that there is no simple universal recipe for a long life.

Unraveling fundamentals of exceptional longevity is essential for the concept of aging; for practical reasons, it may be even more important to get at the mechanisms linking lifespan and health. Theoretical aspects of this problem are reviewed in two articles providing compact but thorough description of the role of mitophagy (Palikaras and Tavernarakis, 2012) and Alu elements (Mustafina, 2013) in aging, while Djansugurova et al. (2013) present new data on genetic markers of cancers. 
Understanding the molecular mechanisms underlying aging and age-associated diseases could bring us closer to the development of novel, efficient, anti-aging treatments. In this regard, one of the latest trends, the use of stem cells to increase lifespan, is described by Kovina et al. (2013).

Recently, there is also a great hope for the development of target-specific drugs for age-associated chronic diseases and, possibly, anti-aging drugs. Several articles in this collection are related to this problem. Vaiserman and Pasyukova (2012) present arguments that the development of specific drugs which target epigenetic pathways could be a highly promising anti-aging strategy. Bharill and co-authors demonstrated that commercially available inhibitors of AKT/FOXO signaling are able to enhance longevity and tolerance to oxidative stress in C. elegans (Bharill et al., 2013). However, the extreme complexity of the genetic control of homeostasis and aging predetermines caution in the use of new drugs. An example of possible side effects of medical treatments is proposed by Smith et al. (2013): the premature and accelerated aging of HIV-patients can be caused by adverse effects of antiretroviral drugs, specifically those that cause severe mitochondrial damage.

Another problem in drug discovery is the difficulty of extrapolating of the results from model species to humans and the time it takes to evaluate the effects of various interventions on longevity in humans. This problem is addressed in the article of Zhavoronkov et al. (2014) who propose a method for screening and ranking the possible geroprotectors before conducting pre-clinical work and expensive clinical trials.

\section{REFERENCES}

Azpurua, J., and Seluanov, A. (2013). Long-lived cancer-resistant rodents as new model species for cancer research. Front. Genet. 3:319. doi: 10.3389/fgene. 2012.00319

Bartke, A., and Westbrook, R. (2012). Metabolic characteristics of long-lived mice. Front. Genet. 3:288. doi: 10.3389/fgene.2012.00288

Bharill, P., Ayyadevara, S., Alla, R., and Shmookler Reis, R. J. (2013). Extreme depletion of PIP3 accompanies the increased lifespan and stress tolerance of PI3K-null C. elegans mutants. Front. Genet. 4:34. doi: 10.3389/fgene.2013. 00034

Coburn, C., and Gems, D. (2013). The mysterious case of the C. elegans gut granule: death fluorescence, anthranilic acid and the kynurenine pathway. Front. Genet. 4:151. doi: 10.3389/fgene.2013.00151

Djansugurova, L. B., Perfilyeva, A. V., Zhunusova, G. S., Djantaeva, K. B., Iksan, O. A., and Khussainova, E. M. (2013). The determination of genetic markers of age-related cancer pathologies in populations from Kazakhstan. Front. Genet. 4:70. doi: 10.3389/fgene.2013.00070

Kovina, M. V., Zuev, V. A., Kagarlitskiy, G. O., and Khodarovich, Y. M. (2013). Effect on lifespan of high yield non-myeloablating transplantation of bone marrow from young to old mice. Front. Genet. 4:144. doi: 10.3389/fgene. 2013.00144
Lehmann, G., Muradian, K. K., and Fraifeld, V. E. (2013). Telomere length and body temperature-independent determinants of mammalian longevity? Front. Genet. 4:111. doi: 10.3389/fgene.2013.00111

Mustafina, O. E. (2013). The possible roles of human Alu elements in aging. Front. Genet. 4:96. doi: 10.3389/fgene.2013.00096

Nazarova, G. G. (2013). Effects of seasonal, ontogenetic, and genetic factors on lifespan of male and female progeny of Arvicola amphibius. Front. Genet. 4:100. doi: 10.3389/fgene.2013.00100

Novoseltsev, V. N., and Novoseltseva, J. A. (2013). Why is individual reproduction in Drosophila flies stochastic? Front. Genet. 3:324. doi: 10.3389/fgene. 2012.00324

Palikaras, K., and Tavernarakis, N. (2012). Mitophagy in neurodegeneration and aging. Front. Genet. 3:297. doi: 10.3389/fgene.2012.00297

Rogina, B., and Helfand, S. L. (2013). Indy mutations and Drosophila longevity. Front. Genet. 4:47. doi: 10.3389/fgene.2013.00047

Sebastiani, P., and Perls, T. T. (2012). The genetics of extreme longevity: lessons from the New England Centenarian study. Front. Genet. 3:277. doi: 10.3389/fgene.2012.00277

Shostal, O. A., and Moskalev, A. A. (2013).The genetic mechanisms of the influence of the light regime on the lifespan of Drosophila melanogaster. Front. Genet. 3:325. doi: 10.3389/fgene.2012.00325

Smith, R. L., deBoer, R., Brul, S., Budovskaya, Y., and van der Spek, H. (2013). Premature and accelerated aging: HIV or HAART? Front. Genet. 3:328. doi: 10.3389/fgene.2012.00328

Trindade, L. S., Aigaki, T., Peixoto, A. A., Balduino, A., Mânica da Cruz, I. B., and Heddle, J. G. (2013). A novel classification system for evolutionary aging theories. Front. Genet. 4:25. doi: 10.3389/fgene.2013.00025

Vaiserman, A. M., and Pasyukova, E. G. (2012). Epigenetic drugs: a novel antiaging strategy? Front. Genet. 3:224. doi: 10.3389/fgene.2012.00224

Yashin, A. I., Arbeev, K. G., Wu, D., Arbeeva, L. S., Kulminski, A., Akushevich, I., et al. (2013). How lifespan-associated genes modulate aging changes: lessons from analysis of longitudinal data. Front. Genet. 4:3. doi: 10.3389/fgene. 2013.00003

Zhavoronkov, A., Buzdin, A. A., Garazha, A. V., Borissov, N. M., and Moskalev, A. A. (2014). Signaling pathway cloud regulation for in silico screening and ranking of the potential geroprotective drugs. Front. Genet. 5:49. doi: 10.3389/fgene.2014.00049

Zimniak, P. (2012). What is the proximal cause of aging? Front. Genet. 3:189. doi: $10.3389 /$ fgene.2012.00189

Conflict of Interest Statement: The authors declare that the research was conducted in the absence of any commercial or financial relationships that could be construed as a potential conflict of interest.

Received: 14 July 2014; accepted: 27 July 2014; published online: 14 August 2014. Citation: Moskalev AA and Pasyukova EG (2014) From theories of aging to anti-aging interventions. Front. Genet. 5:276. doi: 10.3389/fgene.2014.00276

This article was submitted to Genetics of Aging, a section of the journal Frontiers in Genetics.

Copyright (c) 2014 Moskalev and Pasyukova. This is an open-access article distributed under the terms of the Creative Commons Attribution License (CC BY). The use, distribution or reproduction in other forums is permitted, provided the original author(s) or licensor are credited and that the original publication in this journal is cited, in accordance with accepted academic practice. No use, distribution or reproduction is permitted which does not comply with these terms. 